\title{
Ruptured giant intracranial aneurysms. Part I. A study of rebleeding
}

\author{
Vini G. Khurana, M.D., David G. Piepgras, M.D., and Jack P. Whisnant, M.D.
}

Departments of Neurologic Surgery and Health Sciences Research, Mayo Clinic and Mayo Foundation, Rochester, Minnesota

Object. The present study was conducted to estimate the frequency and timing of rebleeding after initial subarachnoid hemorrhage (SAH) from ruptured giant aneurysms.

Methods. The authors reviewed records of 109 patients who suffered an initial SAH from a giant aneurysm and were treated at the Mayo Clinic between 1973 and 1996. They represented 25\% of patients with giant intracranial aneurysms seen at this institution during that 23-year period. Seven of the patients were residents of Rochester, Minnesota, and the rest were referred from other institutions. The aneurysms ranged from 25 to $60 \mathrm{~mm}$ in diameter, and $74 \%$ were located on arteries of the anterior intracranial circulation. The cumulative frequency of rebleeding at 14 days after admission was $18.4 \%$. Cerebrospinal fluid drainage, cerebral angiography, and delayed aneurysm recurrence were implicated in rebleeding in some of the patients. Rebleeding was not precluded by intraaneurysm thrombosis. Among those who suffered recurrent SAH at the Mayo Clinic, 33\% died in the hospital.

Conclusions. Rebleeding from giant aneurysms occurs at a rate comparable to that associated with smaller aneurysms, a finding that should be considered in management strategies.

Key Words * aneurysm * intracranial hemorrhage * rebleeding * subarachnoid hemorrhage

Approximately $5 \%$ of all aneurysms are giant aneurysms, which by definition measure $25 \mathrm{~mm}$ or more in diameter.[27] Approximately $25 \%$ of giant aneurysms present clinically with subarachnoid hemorrhage (SAH).[18,19] Wiebers, et al.,[30] suggested that unruptured giant and near-giant aneurysms have a higher probability of subsequent rupture than smaller ones. Thrombosis of the lesion, which may be extensive in giant aneurysms, does not preclude rupture.[12,22] Although the incidence of rebleeding from smaller aneurysms has been studied extensively, $[5,8,9,14,15,24]$ little is known about the rate of rebleeding from giant lesions. Findings from the Cooperative Aneurysm Study relating to rebleeding from aneurysms of all sizes indicated that the peak interval for rebleeding is the first 24 hours after the initial ictus, when the risk of recurrent SAH is 4.1\%.[8,23] Other investigators have reported rebleeding rates from $0.8[15]$ to $9.6 \%$ [5] during the first 24 hours. The rate thereafter decreases to an average of between $1 \%$ and $2 \%$ per day during the first 14 days, $[8,14,15]$ with a cumulative incidence of between $14.1 \%$ and $26.5 \%$ [8] by the end of the first 2 weeks after the initial hemorrhage. Torner, et al.,[23] stated that aneurysm size is not significantly associated with the risk of recurrent hemorrhage, a view not shared by Paré, et al.[13] 
Because of the sparseness of information related specifically to rebleeding from giant aneurysms, in the present study we examine the frequency of rebleeding and factors that may be associated with recurrent hemorrhage of ruptured giant aneurysms in our patients, who are predominantly a referred population.

\section{CLINICAL MATERIAL AND METHODS}

We reviewed the medical records of all patients with ruptured giant intracranial aneurysms who underwent surgical treatment at the Mayo Clinic between October 1973 and May 1996. Four patients who died of the initial hemorrhage or rebleeding while awaiting surgical treatment for their giant aneurysms were also included in the analysis. This study undoubtedly excluded patients who died of SAH but in whom the diagnosis of ruptured giant aneurysm was not made. The total number of patients with ruptured giant aneurysms in this study was 109 , representing $25 \%$ of all cases of giant aneurysm (ruptured and unruptured) and 5\% of all cases of aneurysm treated surgically at the Mayo Clinic during the study period.

\section{Patient Demographic Data}

Most of the patients (102 or 94\%) included in the present study were referred from other medical centers. The mean age of the patients was 52 years (range 12 weeks-81 years); the majority of patients were in their sixth decade of life. There was a preponderance of female patients, with a female/male ratio of 2:1. A history of hypertension was present in $35 \%$ of all patients.

\section{Diagnostic Details}

For all patients, there was a history of an acute ictus compatible with SAH. Hemorrhage was confirmed by lumbar puncture, computerized tomography (CT) scanning, or surgical findings. The CT scanning was performed in 101 patients and confirmed the presence of SAH in 83\%. Often, the giant aneurysm was demonstrated on CT scanning. With the exception of one patient who had a fatal rehemorrhage at the time of admission and another who died of the effects of the initial hemorrhage before undergoing surgery, cerebral angiographic studies were obtained in all patients and demonstrated an aneurysm in 105 (98\%) of the 107 studies. An aneurysm was not identified angiographically in two patients because of complete thrombosis.

\section{Aneurysm Specifications}

The 109 ruptured giant aneurysms ranged from 25 to $60 \mathrm{~mm}$ in diameter. Eighty-one (74\%) of them were located in the anterior and the other $28(26 \%)$ in the posterior circulation (Table 1). The most common site for a ruptured giant aneurysm (30 patients, 28\%) was the paraclinoid region of the internal carotid artery (ICA). The next most common site (28 patients, 26\%) was the middle cerebral artery. Other sites and characteristics of the aneurysms are summarized in Table 1. Overall, daughter sacs were noted in $7 \%$ of patients, and some degree of intraaneurysm thrombosis was noted in 39\%. Single giant aneurysms were found in 106 patients; each of the other three patients harbored bilaterally symmetrical aneurysms. A combination of multiple giant and nongiant aneurysms was found in $17 \%$ of patients. 


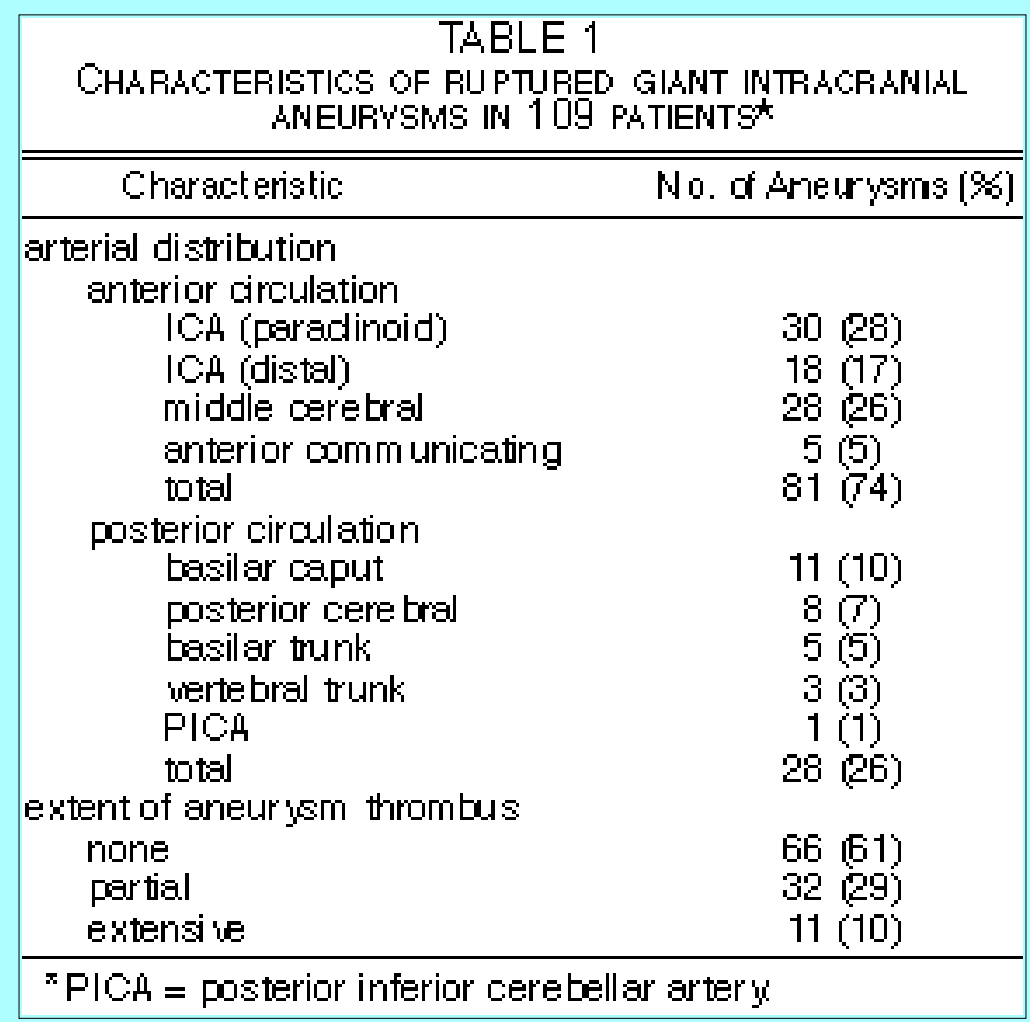

\section{Rebleeding Rate}

Rebleeding was suspected at the time of admission to our institution or thereafter when we observed a sudden change in the patient's neurological status, with an alteration in the sensorium or severity of headache[26] after recovery from the effects of the initial hemorrhage.[14,20] Typically, this was confirmed by lumbar puncture, CT scanning, or surgical findings.

Data from the 109 patients varied with regard to the history of their SAH, including number and dates of previous hemorrhages and time of admission to our institution. Therefore, to simulate as closely as possible a population-based study of rebleeding, we identified a subgroup of 63 patients who were admitted within 7 days of their first hemorrhage from a ruptured giant aneurysm. Data from this subgroup were used to calculate the incidence of rebleeding during the first 2 weeks after admission to our hospital, and life table methods were used to estimate cumulative frequency of rebleeding, with patients being excluded if they died of another cause during the first 2 weeks after admission. To minimize inaccuracy caused by referral bias, [28] the time of admission to our institution rather than the time of initial hemorrhage was used as the reference point (Day 0) in estimating the rebleeding rate.

\section{Follow-Up Evaluation}

Information for follow-up evaluation was obtained primarily from medical records, telephone interviews, and written correspondence with patients, their close relatives, and local physicians. Excluding the 20 patients who died at admission, the interval to the latest medical follow up ranged from 2 weeks to 15 years after discharge, with a mean of 1.7 years and a total follow-up time of 148 patient-years for the study. 


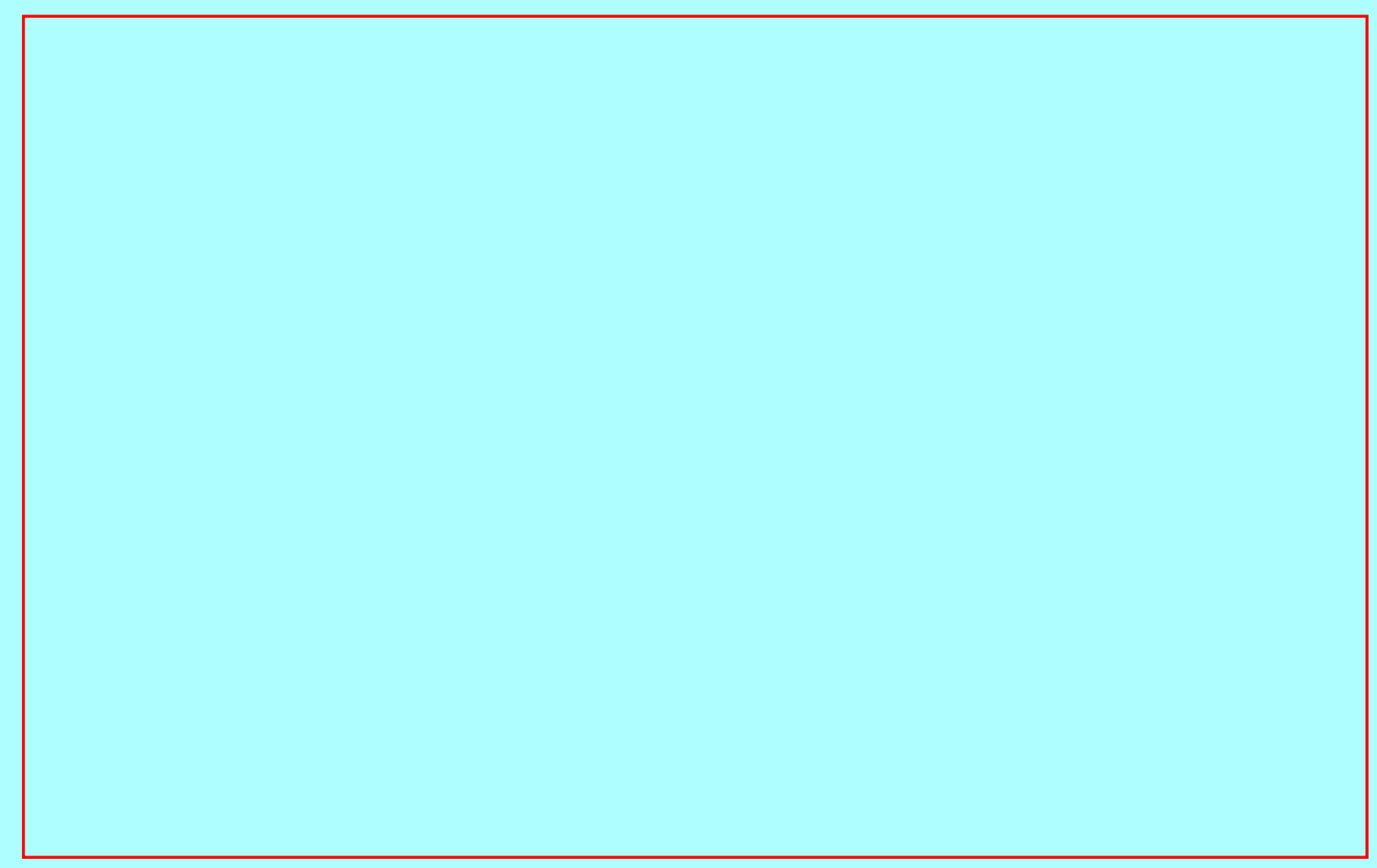

Fig. 1. Bar graph showing time from initial hemorrhage to first rehemorrhage among 31 patients who experienced aneurysm rebleeding. The graph shows data from the entire series of 109 patients. $\mathrm{n} / \mathrm{s}=$ not specified.

\section{RESULTS}

Of the 109 patients, 31 (28\%) suffered an episode of rebleeding at some point during the study: 25 patients presented with one episode of rebleeding, four with two episodes, and two with three episodes. In one of the 31 patients, the date of first rehemorrhage was not specified. The interval between the initial hemorrhage and the first rehemorrhage was less than 15 days in $19(63 \%)$ of the 30 patients in whom the date of first rehemorrhage was known (Fig. 1). Of the 63 patients admitted to our institution within 7 days after their initial hemorrhage, nine (14\%) suffered a first rehemorrhage at some point after admission. In eight of these nine patients, the first rehemorrhage occurred by the end of the 5th day after admission (Fig. 2). 


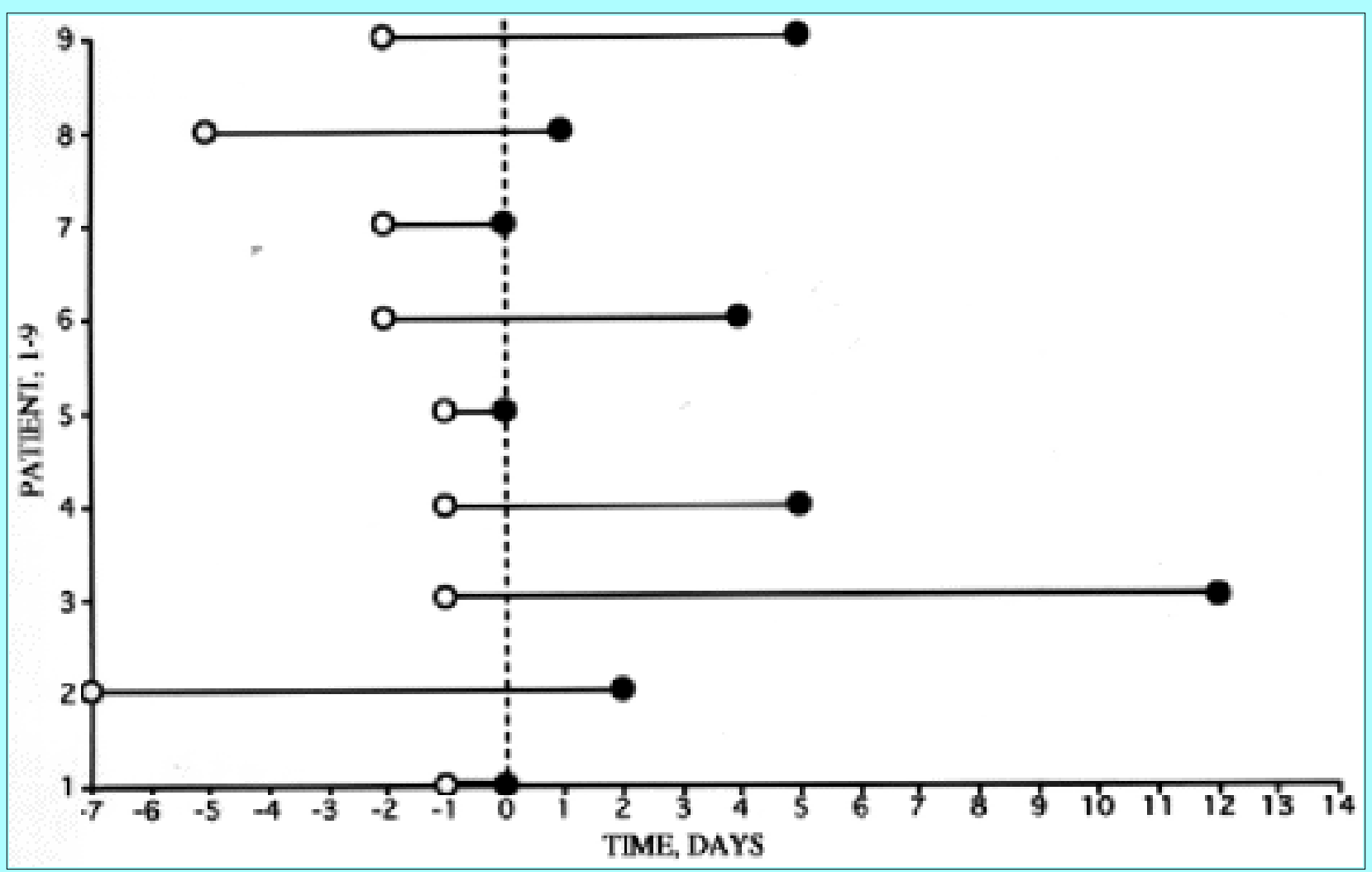

Fig. 2. Distribution plot showing initial hemorrhage and first rehemorrhage relative to the day of admission (Day 0) among patients who experienced an episode of rebleeding at our hospital. The plot shows data from the nine patients in the subgroup of 63 admitted to our institution within 7 days of their first hemorrhage who rebled within the first 2 weeks after admission. Each horizontal line represents the temporal profile of rebleeding for one of the nine patients, from initial hemorrhage (open circle) to first rehemorrhage (closed circle) relative to Day 0 (dotted line).

As shown in Fig. 3, the incidence of rebleeding among these 63 patients from the day of admission was $4.8 \%$ at Day $0,15.7 \%$ at Day 7 , and $18.4 \%$ at Day 14 , with the highest rate of rebleeding found within the first 5 days after admission, before the commencement of the plateau at Day 5. Among the nine patients who rebled in this group, the average time to the first rehemorrhage after admission was 3.4 days, whereas the average time to the first rehemorrhage after the initial SAH was 5.7 days.

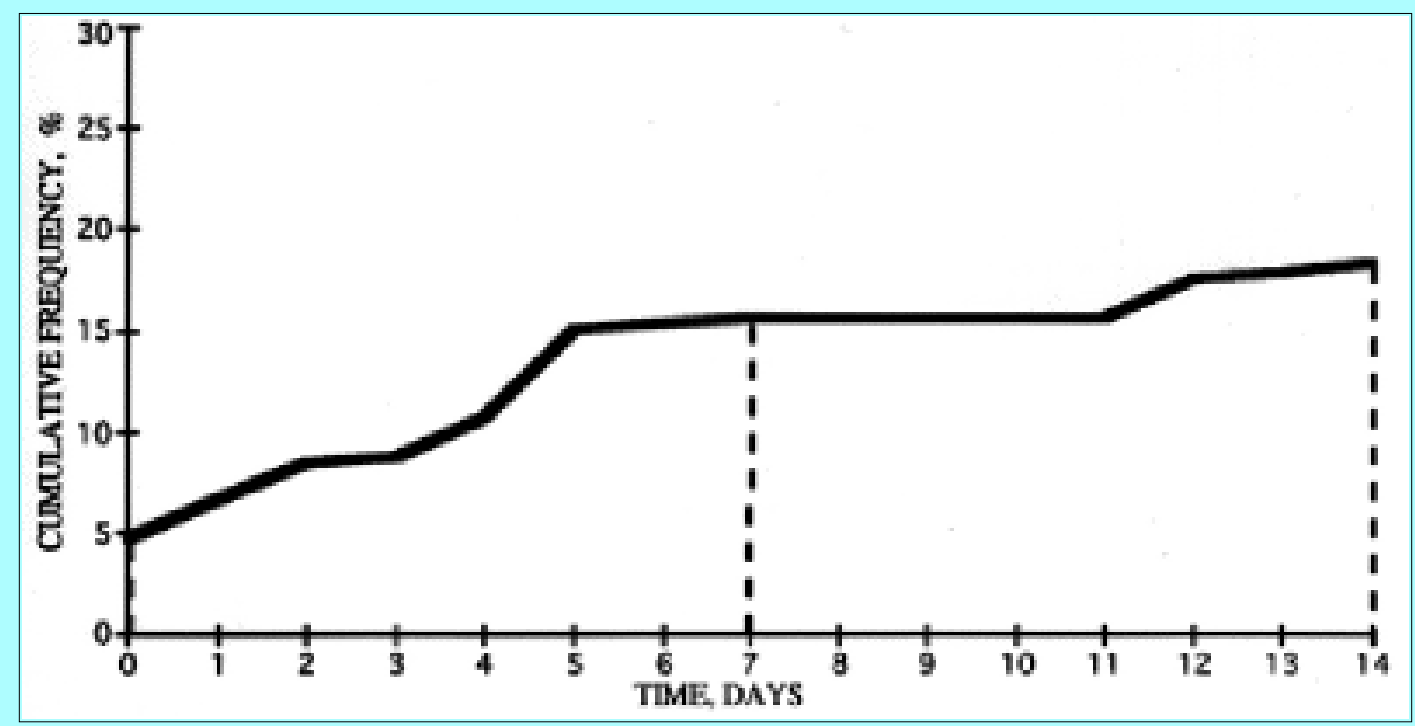


Fig. 3. Graph showing rate of rebleeding after admission (Day 0) for 63 patients admitted to our hospital within 7 days after their first hemorrhage. The cumulative frequency of rebleeding was $4.8 \%$ at Day $0,15.7 \%$ at Day 7 , and $18.4 \%$ at Day 14 .

Thirty-three patients underwent lumbar puncture and one of them experienced an episode of rebleeding shortly after this procedure. None of the four patients who underwent preoperative placement of a ventriculoperitoneal shunt (one patient) or external ventricular drain (three patients) experienced rebleeding after the procedure. One patient in our study suffered a fatal episode of rebleeding immediately after cerebral angiography, and another experienced rebleeding from an aneurysm that had recurred at the site of an apparently successful clipping 8 years previously.

With regard to the relationship between intraaneurysm thrombosis and rebleeding, 13 (30\%) of the 43 patients who had some degree of thrombosis suffered rebleeding. Of these 13 patients, eight exhibited partially thrombosed aneurysms and the other five showed extensively thrombosed aneurysms.

Three of the nine patients who suffered their first rehemorrhage in our hospital died of the effects of the rehemorrhage. Two of these patients died before and the other died soon after surgical treatment.

\section{DISCUSSION}

Several studies have addressed the issue of rebleeding from aneurysms of all sizes. Jane, et al.,[7] reported an incidence of $20 \%$ within the first 14 days after the initial hemorrhage, whereas Phillips, et al.,[14] found a $20 \%$ incidence during the first 10 days. Kassell and Torner[8] noted that the cumulative incidence of rebleeding within the first 2 weeks after the initial hemorrhage was 19\%, with a risk of $14.1 \%$ among patients who had received antifibrinolytic therapy and $26.5 \%$ among those who had not. In a more recent report by Rosenørn, et al.,[15] the 2-week incidence of rebleeding was determined to be $16.8 \%$. In the present study, the cumulative incidence of rebleeding among 63 patients admitted to our hospital within 1 week after their first hemorrhage from a ruptured giant aneurysm was $18.4 \%$ at 14 days after admission. It is essential to recognize that this finding represents the incidence of rebleeding from giant aneurysms in a population of patients composed predominantly of referrals and, for two major reasons, probably underestimates the true incidence of rebleeding in the general population. First, our study excluded those patients with a ruptured giant aneurysm who died of SAH before referral or were not referred because of their poor condition or because they had not undergone angiography to confirm the presence of a giant aneurysm. Second, relatively few patients from the subgroup of 63 were admitted to our hospital within 24 hours after their initial hemorrhage, a period representing the peak interval for rebleeding from aneurysms of all sizes.[5,8] For these reasons, to minimize referral bias,[28] the reference point used to calculate the rebleeding rate was the time of admission to our institution (Day 0) rather than the time of the initial hemorrhage. As a result, our findings reflect the expected rate of rebleeding after admission among patients suffering ruptured giant aneurysms who were referred to experienced neurosurgeons at medical centers similar to our own. Given the finding that among our patients the rate of rebleeding was $18.4 \%$ at 14 days after admission, the absolute rate of rebleeding for ruptured giant aneurysms in the general population is likely to be higher than this and therefore similar to that for smaller aneurysms.

It has been suggested in several studies that unruptured giant and near-giant aneurysms have a higher probability of subsequent rupture than smaller ones,[9,11,30] and some pathological[21] and clinical[13] evidence supports the possibility of a greater risk of rerupture among larger aneurysms. This view is contrary to the findings of Torner, et al.,[23] who stated that the size of an aneurysm is not significantly 
associated with the risk for recurrent hemorrhage. Our findings support the opinion that the rate of rebleeding for giant aneurysms is similar to that for smaller lesions. We have no information about the risks of ultra-early rebleeding in patients with giant aneurysms.

Concerning the risks of aneurysm rebleeding with cerebrospinal fluid drainage, Paré, et al.,[13] reported rebleeding in $2.4 \%$ of patients who underwent lumbar puncture and in $30 \%$ of patients who underwent some form of ventricular drainage. In our study, one (3\%) of the 33 patients who underwent lumbar puncture experienced rebleeding after the procedure, a rate similar to that reported by Paré, et al., although none of the four patients in whom preoperative shunts were placed experienced rebleeding after the procedure.

Tsementzis, et al.,[25] reported that the incidence of cerebral aneurysm rupture during angiography was between $0.02 \%$ and $0.48 \%$. Behr, et al.,[1] reviewed a series of 30 cases of this rare but often fatal complication. In our study, one patient experienced rebleeding immediately after undergoing angiography, a frequency of $0.93 \%$.

With regard to the occurrence of SAH from recurrent giant aneurysms, we found only one example of this among the 105 patients in our series who underwent surgical treatment. Whether the recurrence of this lesion was due to regrowth from a residual neck, refilling after displacement of the original clip, or development of a new aneurysm from the same arterial segment as the first $[3,4,10,16,17]$ could not be determined.

Regarding the relationship between giant aneurysm rupture and intraaneurysm thrombosis, growth or rupture of such aneurysms is not precluded by either partial or complete thrombosis.[2,6,12,22,29] Similarly, rebleeding is not precluded by the presence of thrombus within a giant aneurysm, as indicated by our finding that 13 of the 43 patients in our study who showed some degree of intraaneurysm thrombosis experienced an episode of rebleeding.

In two early series, Locksley[9] and Trumpy[24] reported a mortality rate of approximately $40 \%$ for first rehemorrhages for the entire range of aneurysm size. We observed a 33\% mortality rate among patients with giant aneurysms who experienced their first rehemorrhage at our hospital.

\section{CONCLUSIONS}

The principal finding of the present study is that among patients referred to our institution, rebleeding of giant aneurysms occurred at a rate comparable to that seen in smaller lesions. Rebleeding, which was associated with a mortality rate of $33 \%$, was not precluded by the presence of intraaneurysm thrombus and could not be strongly linked to interventions such as cerebrospinal fluid drainage or cerebral angiography. We believe that neurosurgeons should consider these findings when developing management strategies for patients with ruptured giant aneurysms.

\section{References}

1. Behr R, Agnoli AL, Zierski J: Rupture of giant cerebral aneurysms during angiography. Case report and review of literature. J Neurosurg Sci 32:195-202, 1988

2. Drake CG: Giant intracranial aneurysms: experience with surgical treatment in 174 patients. Clin Neurosurg 26:12-95, 1979 
3. Fujiwara S, Fujii K, Fukui M: De novo aneurysm formation and aneurysm growth following therapeutic carotid occlusion for intracranial internal carotid artery (ICA) aneurysms. Acta Neurochir 120:20-25, 1993

4. Giannotta SL, Litofsky NS: Reoperative management of intracranial aneurysms. J Neurosurg 83:387-393, 1995

5. Hillman J, von Essen C, Leszniewski W, et al: Significance of "ultra-early" rebleeding in subarachnoid hemorrhage. J Neurosurg 68:901-907, 1988

6. Hosobuchi Y: Direct surgical treatment of giant intracranial aneurysms. J Neurosurg 51:743-756, 1979

7. Jane JA, Winn HR, Richardson AE: The natural history of intracranial aneurysms: rebleeding rates during the acute and long term period and implication for surgical management. Clin Neurosurg 24:176-184, 1977

8. Kassell NF, Torner JC: Aneurysmal rebleeding: a preliminary report from the Cooperative Aneurysm Study. Neurosurgery 13:479-481, 1983

9. Locksley HB: Natural history of subarachnoid hemorrhage, intracranial aneurysms and arteriovenous malformations. Report of the Cooperative Study of Intracranial Aneurysms and Subarachnoid Hemorrhage. Section V, Part II. Based on 6368 cases in the Cooperative Study. J Neurosurg 25:321-368, 1966

10. Maiuri F, Spaziante R, Iaconetta G, et al: 'De novo' aneurysm formation: report of two cases. Clin Neurol Neurosurg 97:233-238, 1995

11. McCormick WF: Problems and pathogenesis of intracranial arterial aneurysms, in Moossy J, Janeway R (eds): Cerebral Vascular Diseases. Seventh Conference on Cerebral Vascular Diseases, 1970, Princeton. New York: Grune \& Stratton, 1971, pp 219-231

12. Nagahiro S, Takada A, Goto S, et al: Thrombosed growing giant aneurysms of the vertebral artery: growth mechanism and management. J Neurosurg 82:796-801, 1995

13. Paré L, Delfino R, Leblanc R: The relationship of ventricular drainage to aneurysmal rebleeding. J Neurosurg 76:422-427, 1992

14. Phillips LH II, Whisnant JP, O'Fallon WM, et al: The unchanging pattern of subarachnoid hemorrhage in a community. Neurology 30:1034-1040, 1980

15. Rosenørn J, Eskesen V, Schmidt K, et al: The risk of rebleeding from ruptured intracranial aneurysms. J Neurosurg 67:329-332, 1987

16. Sakaki T, Takeshima T, Tominaga M, et al: Recurrence of ICA-PCoA aneurysms after neck clipping. J Neurosurg 80:58-63, 1994

17. Samejima H, Mizokami T, Ushikubo Y, et al: [Studies of recurrence of cerebral aneurysm after operation.] No Shinkei Geka 12:873-881, 1984 (Jpn)

18. Sengupta RP: Management of large and giant aneurysms. Neurosurg Rev 5:173-178, 1982 
19. Sundt TM Jr, Piepgras DG: Surgical approach to giant intracranial aneurysms. Operative experience with 80 cases. J Neurosurg 51:731-742, 1979

20. Sundt TM Jr, Whisnant JP: Subarachnoid hemorrhage from intracranial aneurysms. Surgical management and natural history of disease. N Engl J Med 299:116-122, 1978

21. Suzuki J, Ohara H: Clinicopathological study of cerebral aneurysms. Origin, rupture, repair, and growth. J Neurosurg 48:505-514, 1978

22. Swearingen B, Heros RC: Fatal rupture of a thrombosed giant basilar artery aneurysm. Surg Neurol 23:299-302, 1985

23. Torner JC, Kassell NF, Wallace RB, et al: Preoperative prognostic factors for rebleeding and survival in aneurysm patients receiving antifibrinolytic therapy: report of the Cooperative Aneurysm Study.

Neurosurgery 9:506-513, 1981

24. Trumpy JH: Subarachnoid haemorrhage. Time sequence of recurrences and their prognosis. Acta Neurol Scand 43:48-60, 1967

25. Tsementzis SA, Kennett RP, Hitchcock ER: Rupture of intracranial vascular lesions during arteriography. J Neurol Neurosurg Psychiatry 47:795-798, 1984

26. Verweij RD, Wijdicks EFM, van Gijn J: Warning headache in aneurysmal subarachnoid hemorrhage: a case-control study. Arch Neurol 45:1019-1020, 1988

27. Weir B: Aneurysms Affecting The Nervous System. Baltimore: Williams \& Wilkins, 1987, pp $187-208$

28. Whisnant JP, Sacco SE, O'Fallon WM, et al: Referral bias in aneurysmal subarachnoid hemorrhage. J Neurosurg 78:726-732, 1993

29. Whittle IR, Dorsch NW, Besser M: Spontaneous thrombosis in giant intracranial aneurysms. J Neurol Neurosurg Psychiatry 45:1040-1047, 1982

30. Wiebers DO, Whisnant JP, Sundt TM Jr, et al: The significance of unruptured intracranial saccular aneurysms. J Neurosurg 66:23-29, 1987

Manuscript received January 30, 1997.

Accepted in final form October 1, 1997.

This study was supported in part by a scholarship from the Sydney Tapping Bequest, Postgraduate Committee in Medicine, The University of Sydney, Sydney, New South Wales, Australia.

Address reprint requests to: David G. Piepgras, M.D., Mayo Clinic, 200 First Street SW, Rochester, Minnesota 55905. 